\title{
Surgical Treatment of Intratemporal Facial Nerve Tumors
}

\author{
Kh. M. Diab, N. A. Daikhes, I. I. Nazhmudinov, D. S. Kondratchikov, \\ O. A. Pashchinina, A. S. Korobkin, O. S. Panina \\ Federal Research Clinical Center of Otorhinolaryngology of \\ Federal Medical Biological Agency of Russia, \\ (Director - Professor N. A. Daikhes), 123182, Moscow, Russia
}

\begin{abstract}
Purpose: To report the management, surgical procedure and outcomes from a case series of intratemporal facialnerve tumors. The management of patients with intratemporal tumors of the facial nerve has progressively evolved from performing microsurgical excision with restoration of the facial nerve to more conservative techniques aiming at preserving the integrity of the facial nerve.

Methods: Sixteen patients with facial nerve tumors underwent surgery. The surgical approach was proposedafter a first phase of expectant management with careful observation of symptoms evolution. After tumor removal, a facio-facial nerve suture was performed in three cases. For the other cases, an intermediate graft from the great auricular nerve or the sural nerve was used.

Results: A direct neurorrhaphy and plastics with intermediate graft allowed improvement of the facial functionby one grade on the House-Brackmann scale. During the observation period, which covered on average 10 months $\left({ }^{+} / 5\right.$ months), tumor recurrence was not detected in any of the 16 patients. This was confirmed by post-surgical MRI examination

Conclusions: In spite of the fact that this pathology is quite rare, it is a priority disease because of its importantsocial and functional meaning. Paresis and mimic muscles paralysis is a great tragedy for a patient, and in this case the priority is to safe facial nerve functions. The efficiency of surgical treatment of facial nerve's tumor and its functions' restoration directly depends on its timely definition based on modern radiologic examination, prevalence rate of tumor's stage and time duration of paresis.
\end{abstract}

Keywords (4-6): facial nerve, neuroma, facial paresis, schwannoma.

\section{Introduction}

Among the affections potentially involving the VII ${ }^{\text {th }}$ pair of cranial nerves or facial nerve $(\mathrm{FN})$, and causing signs of facial hemiparesis, primary FN tumors are relatively rare.

Bell's palsy is the most common affection of the FN, although it's causes are still not well understood, it is characterized by a transient unilateral facial muscle weakness, leading to facial paralysis in most severe cases and accompanied by taste modifications, and transient hyperacusis. Bell's palsy usually recovers spontaneously in period from 14 days to 6 months [1]. Besides this common affection, facial paralysis can also develop from various pathological conditions affecting the postcranial fossa, temporal bone, parotid gland or infratemporal fossa. In particular, secondary involvement of the FN can be caused by traumatic injuries of the infratemporal fossa, or by the presence of erosive and/or expansive formations such as cholesteatoma, squamous cell carcinoma, glomus tumors, acoustic neuroma, meningioma, and malignant neoplasms of the parotid gland. Rare cases of malignant epithelioid tumors originating from the cranial nerves' sheath and involving the trigeminal nerve and FN were also described [2$3]$. However, most of the malignant tumors of the 
FN reported are the result of metastases or perineural invasions resulting from distant primary tumors such as breast, lung or kidney cancers, often with a metastases spread into the internal auditory canal [4-5].

When considering primary tumors of the FN, neurinoma and hemangioma are the most commonly observed, whereas neurofibroma, granular cell tumors, meningioma, and primary glomus tumors are less common [6-7]. The nomenclature of neurogenic tumors of the FN includes neurinoma, schwannoma and neurilemmoma. Histologically, these tumors are of neuroectodermal origin, contrarily to neurofibroma, which develop from endoneural connective tissues and are more commonly observed in the context of the neurofibromatosis disease. Facial nerve Schwannomas (FNSH) are rare, slowly growing tumors, representing less than $1 \%$ of all cases of temporal bone tumors. They are typically single, unilateral and naturally sporadic tumors [6-8], without any marked gender asymmetry [7]. FNSH are benign, encapsulated tumors, arising from Schwann cells. In certain cases, intratumoral cystic developments or hemorrhages can be observed [7-9]. The microscopic evaluation of the tumor reveals the diffuse aspect of the thickened sheath of the FN. FNSH can develop at any site along the VII ${ }^{\text {th }}$ cranial nerve, starting from its origin at the cerebellopontine angle, to its upper posterior auricular branch, along its extra-temporal course, passing through the parotid gland. Most often the tumor will affect the tympanic segment of the FN, less frequently the segment of the geniculate ganglion / labyrinth, or the mastoid segment, large tumors can also cover several segments [6]. Malignant FNSH remain extremely rare [10]. Symptoms can vary slightly depending on the extent and exact location of the tumor, but FNSH usually presents as a peripheral neuropathy with or without otological symptoms, including conductive and sensorineural hearing loss [7-9, 11].

The paralysis of facial muscles can be absent initially and will most often develop progressively, with a first phase of muscular weakness evolving to a paralysis. This progressive character is due to the relative tolerance of the neural tissues to this kind of tumors, caused by an extreme slow growth and abundant vascularization [12]. Sometimes FNSH can develop inside the tissue of the parotid salivary gland. Extremely rarely schwannomas can affect the peripheral branches of the FN at different levels at the same time $[9,11]$. In order to diagnose FN tumors, high resolution CT (HRCT) with a high-spatial-frequency (bone) reconstruction algorithm is mandatory. Depending on the scanning method or on the reconstruction algorithm chosen, the FN neuroma, usually framed by the internal acoustic canal and the fallopian canal, might not show evident contrast differences with bony tissues and may therefore be hard to visually delineate with precision. Therefore MRI with a contrast agent is a more sensitive method to detect FN tumors that are in close contact with the internal acoustic canal. At this stage without any facial paresis, it is very difficult to ascertain that the tumor is indeed originating from the FN, rather than from the cochleo-vestibular nerve bundle for example. Often, acoustic neuroma are located closer to the center of the internal acoustic canal. FN hemangiomas show abnormal, blurred or vague boundaries on HRCT, which can differentiate them form schwannomas which usually present clearer margins. Moreover, the bone tissues can show spicules near a FN hemangioma on the CT scan that are not observed for schwannomas [13]. This can be attributed to an osteoclast mediated remodeling of the bone tissue in the affected area, forming lamellate trabecular bone [14].

The use of electromyography is also very important for complex diagnostics in patients presenting facial paralysis. Electromyography allows to precisely quantify the contractility of facial muscles, thereby offering an indirect measure of the severity of the degenerative changes occurring in the muscles and in the FN, and provides some measures of the conductivity of nerve fibers along the different FN branches [15]. Large tumors can grow up and expose the dura mater of the middle and posterior cranial fossae and penetrate inside the brain with potential development of intracranial complications. The tumor can also evolve inside the parotid gland after the destruction of the anterior wall of the external auditory canal and soft tissues of the neck can be affected as well following the destruction of the apex of the mastoid process. The surgical procedure of FN schwannoma today consists in realizing a full resection of the tumor while aiming at a conservation or reconstruction of facial function for a long period of time. 


\section{Patients}

From December 2014 to March 2017, 16 patients were treated for FN tumors in our clinic. All underwent full ENT examination, including endoscopic otoscopy (Fig. 1). Audiological examination included pure-tone threshold audiometry over the standard frequency range and tuning-fork tests. House-Brackmann scale was used to assess the severity of FN dysfunction [16]. CT and MRI were performed for diagnosis and to precisely determine the localization and extension of the tumor. Electromyographic examination was conducted on each patient preoperatively in order to assess the conduction of neural impulses along the peripheral branches of the FN.

\section{Surgical resection of Facial Nerve schwannoma}

In case of preserved facial musculature function, surgical intervention was performed under facial nerve monitoring, using a post-auricular approach. When the tumor was localized in the mastoid segment of the FN, an antromastoidotomy was performed, with an enlargement of the access depending on the extent of the tumor. The posterior wall of the external auditory canal was removed if the tympanic segment or the geniculate ganglion was involved.

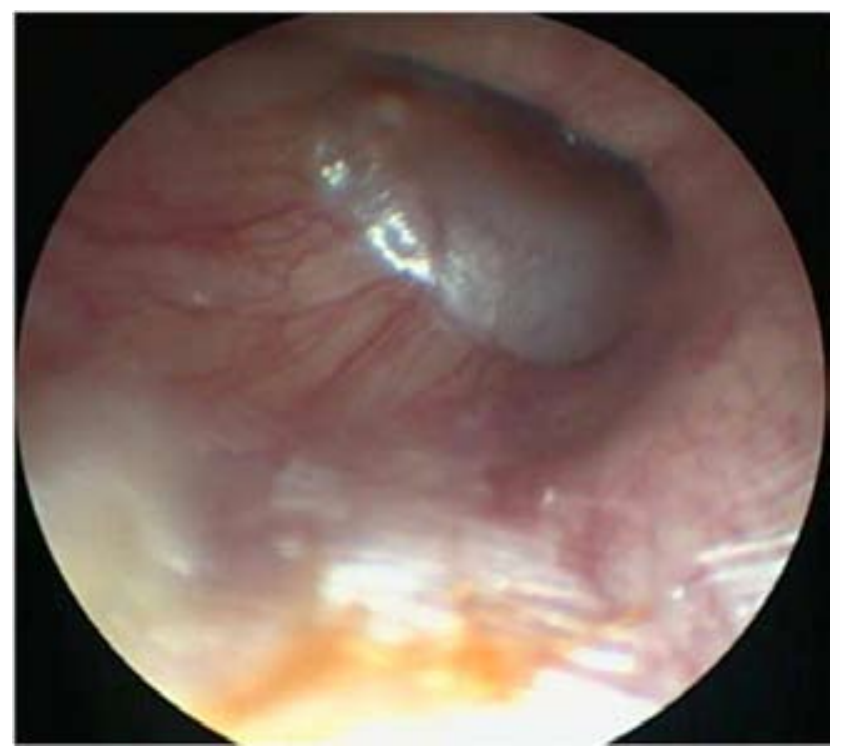

Fig. 1. Otoscopic picture of the proliferating facial nerve schwannoma over the mastoid and tympanic FNsegments, right ear image.

When possible, the tumor was removed by resecting it from the main trunk of the facial nerve with a careful anatomical dissection (Fig. 2). In case of invasive growth of the tumor inside the nerve's sheath, an in the general case of an abnormal appearance of the nerve structure, the tumor was dissected together with the affected nerve portion. A reconstructive neural surgery was then performed, either when the traumatic zone was not too extended, by performing a direct neurorrhaphy of the facial nerve by suturing the nerve stumps after meticulous alignment. In cases for which the direct facial-facial nerve suture was impossible, an interpositional graft was used to obtain a tension-free nerve suture (Fig. 3). In one case presenting a long anamnesis of facial muscles paralysis ( 9 years), no facial nerve reconstruction was performed. When the auditory ossicles were implicated in the tumor extensions, they were removed and a tympanoplasty and ossiculoplasty using titanium middle-ear prostheses were performed. At the final stage of the surgery, a myringoplasty using a temporalis fascia was performed.

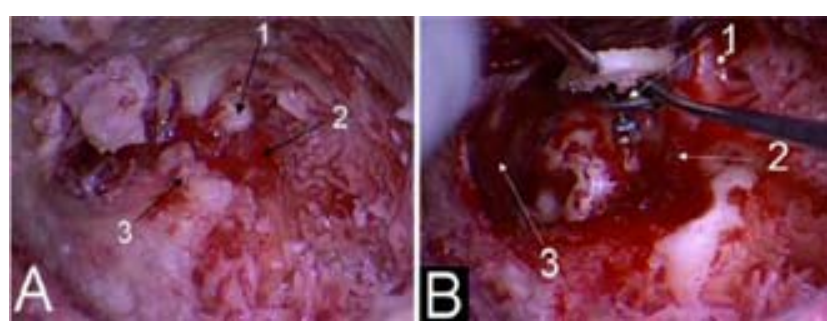

Figure 2. Intraoperative photography of a left ear surgery. A - tumor removal stage, where 1 is the incus, 2 is thetumor, and 3 is the external auditory canal. B reconstruction stage, where 1 - stapes and partial titanium prosthesis, 2 - tympanic segment of the facial nerve, 3 - auto-facial flap.

In order to evaluate the outcomes of the surgical procedure, functional (hearing and degree of mimic paresis) and anatomical results (imaging) were evaluated after long-term post-op follow-up observation. Anatomical outcomes were considered as positive in the absence of a tumor recurrence as evaluated by MRI and CT imaging and by the visual aspect of the tympanoplasty. Auditory function was assessed by analyzing the post-op pure-tone threshold audiograms, considering bone and air conduction thresholds as well as the estimation of the air-bone gap (ABG). Criteria for positive functional outcomes were a lowering of the air conduction thresholds by $20 \mathrm{~dB}$ HL or less and a reduction of the ABG by $15 \mathrm{~dB}$ HL. 

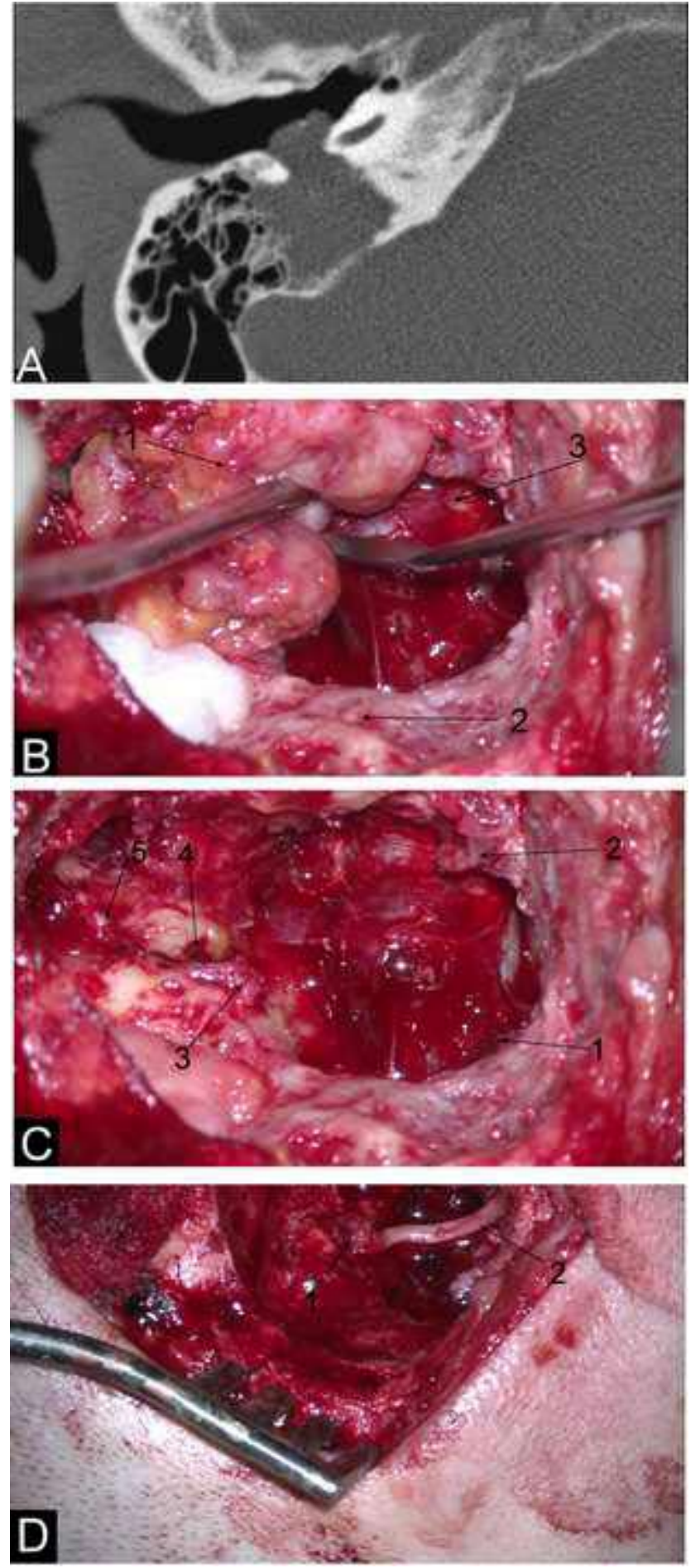

Fig. 3. Removal of FNSH (right ear). A - computer tomography of the right temporal bone, axial projection. B -stage of tumor removal, where 1 - tumor, 2 - bone wall of sigmoid sinus, 3 - internal carotid artery. B - general view of the cavity after complete removal of the tumor, where 1 - the bulb of the internal jugular vein, 2 - the distal end of the facial nerve, 3 - proximal end of FN, 4 - round window, 5 - stapes. D - final form of the trepanation cavity after reconstruction of the facial nerve, where 1 - proximal anastomosis, 2 - autograft $n$. Suralis

\section{Results and Discussion}

The management of intratemporal tumors of the FN has evolved from microsurgical resection with restoration of the nerve to more conservative techniques [15, 17-18]. The more recent approach includes an expectant management with careful observation in order to monitor the slow growth of the tumor followed by decompression of the nerve fibers or stereotactic radiosurgery. The surgical resection of the $\mathrm{FN}$ is a controversial issue when the function of mimic muscles is normal or close to normal (1-2 degrees on House-Brackmann scale) [17-19]. When the diagnosis, either by visual inspection of pre-op images or by electrophysiological examination, suggests the presence of a highly probable FN neurogenic tumor, then the priority is to protect the functionality of the FN or even to improve it through surgical intervention, regardless of the size of the tumor [20-21]. The exception to this principle being when the tumor is causing a compression of the brainstem or an erosion of the bony labyrinth. The final choice of treatment is based on the intention to preserve the FN function. The consensus today is that surgery should be used when patients show signs of worsening of clinical and radiological conditions (paralysis stage III to IV on House-Brackmann scale, and radiological signs of growth or risk of complications). In this context it is worth mentioning that the chances of positive outcomes and functional results after surgical intervention decrease considerably if the resection is performed later than the first year since the beginning of the FN dysfunction [22].

In the current study, patients were observed for a period of four to 20 months post-surgery. Table 1 shows the localization, histological characteristics of tumors, type of surgical interference, and the grade of hearing loss and facial paralysis at preand postoperative periods. 


\begin{tabular}{|c|c|c|c|c|c|c|c|c|c|}
\hline \multicolumn{2}{|c|}{ 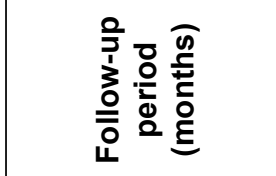 } & \multirow{2}{*}{$\frac{\stackrel{\rho}{\leftarrow}}{\sim}$} & \multirow{2}{*}{$\begin{array}{l}\stackrel{\varphi}{\circ} \\
\infty\end{array}$} & \multirow{2}{*}{$\begin{array}{l}\stackrel{0}{\sigma} \\
+\end{array}$} & \multirow{2}{*}{$\stackrel{\stackrel{N}{\sim}}{\infty}$} & \multirow{2}{*}{$\frac{\infty}{\infty}$} & \multirow{2}{*}{$\begin{array}{l}\infty \\
\infty\end{array}$} & \multirow{2}{*}{\begin{tabular}{|l}
$\infty$ \\
$\infty$
\end{tabular}} & \multirow{2}{*}{\begin{tabular}{|l} 
\\
0
\end{tabular}} \\
\hline סِ d & $\frac{\bar{\Phi}}{\frac{ \pm}{\sigma}}$ & & & & & & & & \\
\hline Z & $\begin{array}{l}0 \\
\frac{0}{0} \\
\frac{0}{0}\end{array}$ & $\sim$ & 0 & 10 & - & $\nabla$ & 0 & 0 & 0 \\
\hline \multirow{2}{*}{ 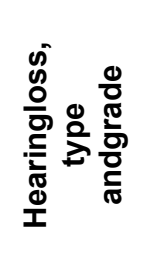 } & $\stackrel{\bar{\Phi}}{\frac{\Phi}{\sigma}}$ & $\begin{array}{l}\overline{\dot{T}} \\
\stackrel{\bar{c}}{\omega}\end{array}$ & $\bar{\Sigma}$ & $\begin{array}{l}\overline{\mathbb{0}} \\
\sum_{0}^{\circ} \\
\text { z }\end{array}$ & $\bar{\Sigma}$ & $\begin{array}{l}\overline{0} \\
\sum_{0}^{\circ} \\
z\end{array}$ & $\bar{\Sigma}$ & $\bar{\Sigma}$ & $\bar{\Sigma}$ \\
\hline & ڤั) & $\equiv$ & $\begin{array}{l}\bar{\sigma} \\
\sum_{0}^{\circ} \\
z\end{array}$ & $\begin{array}{l}\overline{\tilde{\sigma}} \\
\stackrel{5}{0} \\
\text { ż }\end{array}$ & $\equiv$ & $\begin{array}{l}\overline{\mathrm{\sigma}} \\
\sum_{\overline{0}}^{\circ} \\
z\end{array}$ & $\bar{\Sigma}$ & $\begin{array}{l}= \\
0\end{array}$ & $\begin{array}{l}= \\
0\end{array}$ \\
\hline \multicolumn{2}{|c|}{$\begin{array}{l}\frac{3}{0} \\
\text { o } \\
\stackrel{5}{5}\end{array}$} & 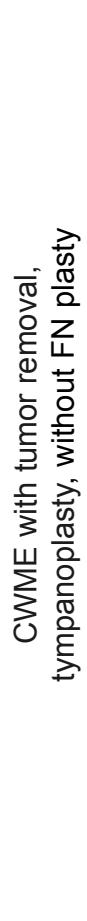 & 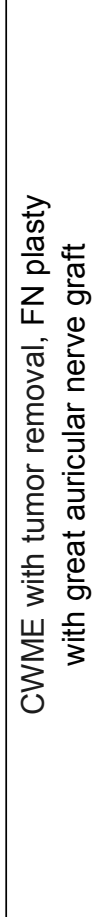 & 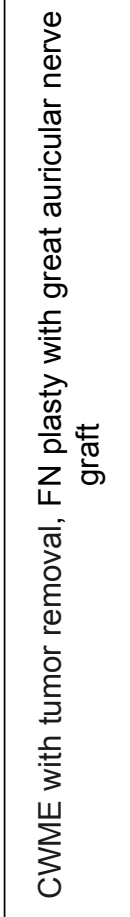 & 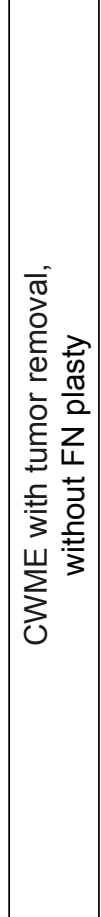 & 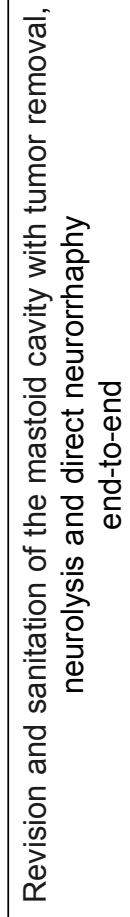 & 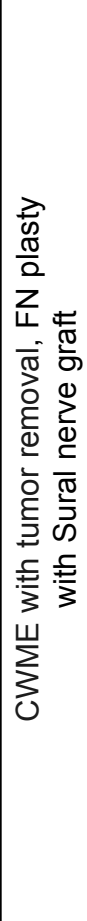 & 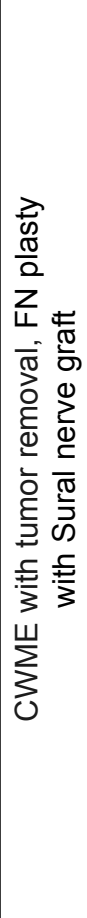 & 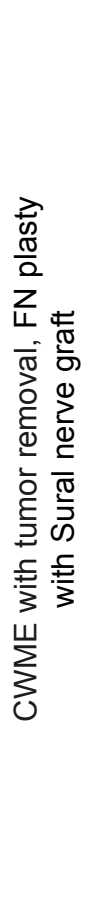 \\
\hline \multicolumn{2}{|c|}{ 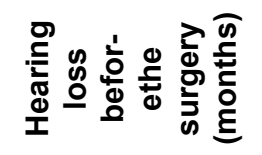 } & ' & $\sim$ & $\sim$ & ' & $\stackrel{m}{=}$ & $\nabla$ & مصا & ما \\
\hline \multicolumn{2}{|c|}{ 高离 } & $\vdash$ & $\Sigma$ & $\Sigma$ & $\begin{array}{l}0 \\
\text { D } \\
+ \\
+ \\
+ \\
亡\end{array}$ & $\Sigma$ & $\Sigma$ & $\Sigma$ & $\Sigma$ \\
\hline \multicolumn{2}{|c|}{ 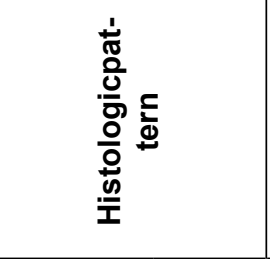 } & $\begin{array}{l}\stackrel{0}{\varepsilon} \\
\frac{0}{0} \\
\frac{0}{2} \\
z\end{array}$ & 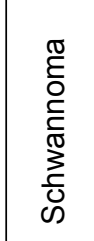 & 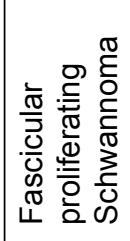 & 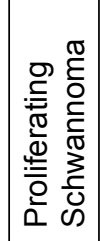 & 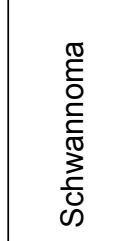 & 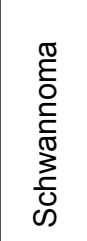 & 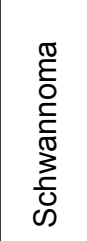 & 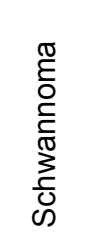 \\
\hline \multicolumn{2}{|l|}{$\stackrel{\circ}{\mathbf{z}}$} & - & $N$ & $m$ & $\nabla$ & ما & 0 & $r$ & $\infty$ \\
\hline
\end{tabular}




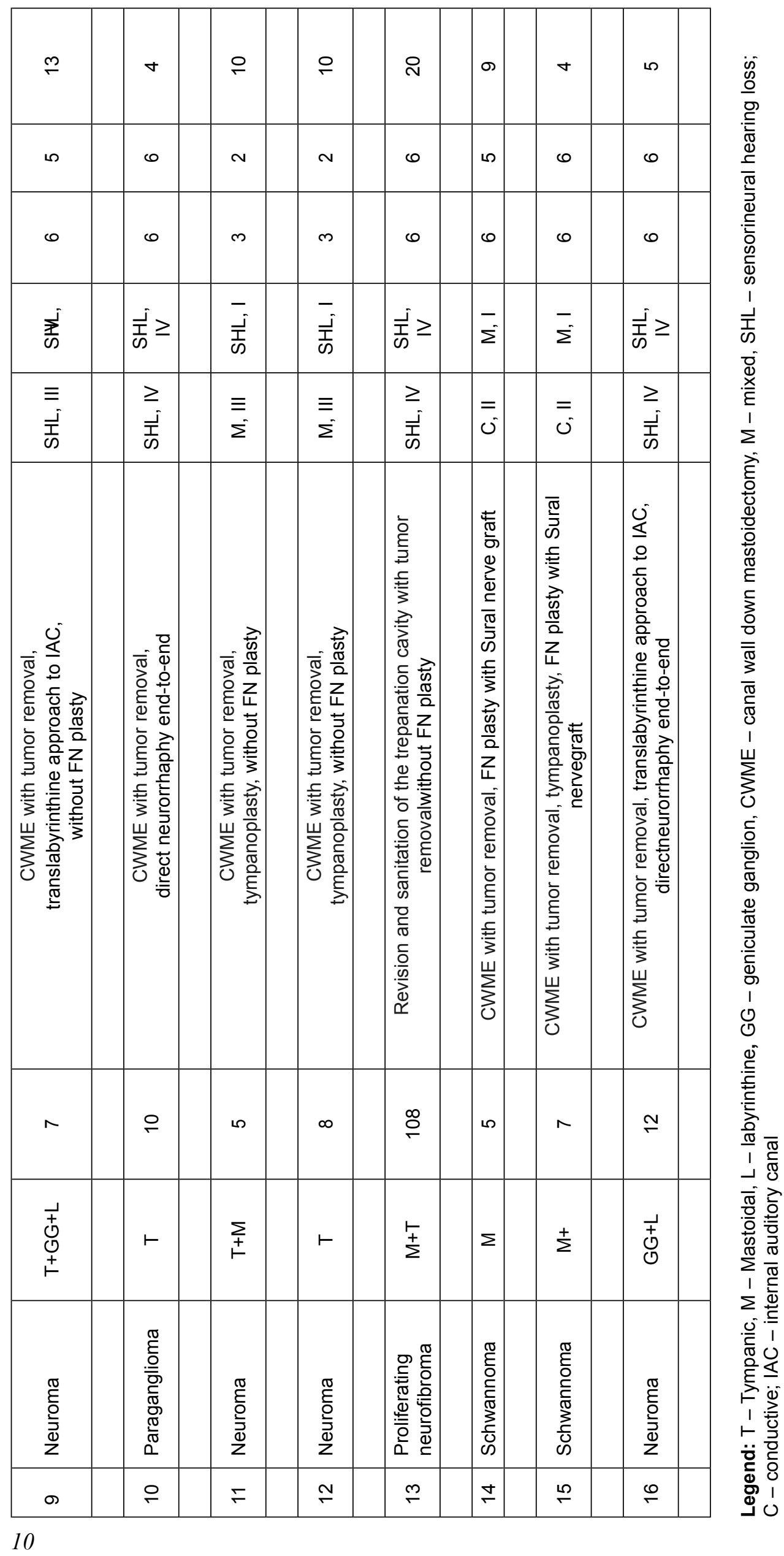


Simultaneous reconstruction of the sound-conducting system and the tumor resection were performed for four patients with mixed hearing loss in the preoperative period. The thresholds of air conduction decreased by $25 \mathrm{~dB} \mathrm{HL}$, and the ABG decreased approximately to $15 \mathrm{~dB}$ HL. The middle-ear ossicular chain was not involved in four patients who showed hearing thresholds in the normal range preoperatively. Direct neurorrhaphy and facial nerve reconstruction with interposition graft allowed to improve facial muscles function by one HouseBrackmann grade in six different patients. In four cases, and despite reconstruction neurosurgery, the function of the mimic muscles could not be improved. This observation could be explained by a short period of postoperative observation, and/or by a prolonged history of facial paralysis, with a partial atrophy of the facial muscles. For the indicated surveillance period, no recurrence of tumors in any patient was observed. This was confirmed by post-op MRI observations and during revision surgeries (in two cases). Irradiation is an alternative method for patients with early facial dysfunction (II-III stages according to House- Brackmann scale) and documented worsening clinical and radiological conditions. This procedure has relatively small but significant risk of causing a hearing loss and/or mimic muscles dysfunction and should be discussed with the patient before the decision to undertake such a treatment is made [23-26].

\section{Conclusion}

Despite the fact that $\mathrm{FN}$ schwannomas is a quite rare condition, it is a priority disease because of its important social and functional impact. Facial hemiparesis and mimic muscles paralysis is a great tragedy for one patient, and in this case the priority is to maintain facial nerve functions. The efficiency of surgical treatment of facial nerve's tumor and its functions' restoration directly depends on its timely diagnosis and decision to intervene, based on modern radiologic examination, prevalence rate of tumor's stage and duration of paresis.

Disclosure of interest. The authors declare that they have no conflicts of interest concerning this article.

\section{References}

1. McAllister K, Walker D, Donnan PT, Swan I (2013) Surgical interventions for the early management of Bell's palsy. CochraneDatabaseSystRev 10:CD007468.

2. Fisher BJ, Dennis KE (2006) Malignant epithelioid cranial nerve sheath tumor: case report of a radiation response. J Neurooncol 78:173177.

3. Bowers CA, Taussky P, Duhon BS, Chin SS, Couldwell WT (2011) Malignant peripheral nerve sheath tumour of the trigeminal nerve: case report and literature review. Br J Neurosurg 25:750-753.

4. Barrera-Flores FJ, Villarreal-Del Bosque N, Dнаz Gonzбlez-Colmenero A et al (2017) Perineural spread-susceptible structures: a non-pathological evaluation of the skull base. EurArchOtorhinolaryngol 274:2899-2905.

5. Stambuk HE (2013) Perineural tumor spread involving the central skull base region. SeminUltrasound CT MR 34:445-58.

6. Diab Kh., Najmudinov I.I.,Karayan A.S., Kondratchikov D.; Pashchinina O., Orlova E., Usifov K. Sugical treatment of intratemporal tumors of facial nerve. Vrach (The Doctor).2018 P 49-54. (In Russ)

7. Ross L, Drazin D, Eboli P, Lekovic GP (2013) Atypical tumors of the facial nerve: case series and review of the literature. NeurosurgFocus 34:E2.

8. Kim CS, Chang SO, Oh SH, Ahn SH, Hwang CH, Lee HJ (2003) Management of intratemporal facial nerve schwannoma. OtolNeurotol 24:312-316

9. Kirazli T, Oner K, Bilgen C, Ovul I, Midilli R (2004) Facial nerve neuroma: clinical, diagnostic, and surgical features. SkullBase 14:115120.

10. O’Donoghue GM, Brackmann DE, House JW, Jackler RK (1989) Neuromas of the facial nerve. AmJ Otol 10:49-54.

11. Gogate BP, Anand M, Deshmukh SD, Purandare SN (2013) Malignant peripheral nerve sheath tumor of facial nerve: Presenting as parotid mass. J OralMaxillofacPathol 17:129-131.

12. Passos IM, Massuda ET, Hyppolito MA, Colli BO, Damico TA (2015) Rare case of neurinoma of the facial nerve. Braz J Otorhinolaryngol $81: 226-227$

13. Wilkinson EP, Hoa M, Slattery WH III ${ }^{\text {rd }}$, et al (2011) Evolution in the management of facial nerve schwannoma. Laryngoscope 121:20652074.

14. Mundada P, Purohit BS, Kumar TS, Tan TY (2016) Imaging of facial nerve schwannomas: diagnostic pearls and potential pitfalls. DiagnIntervRadiol 22:40-46.

15. Mijangos SV, Meltzer DE (2011) Case 171: facial nerve hemangioma. Radiology 260:296-301.

16. Volk GF, Pantel M, Guntinas-Lichius O (2010) Modern concepts in facial nerve reconstruction. HeadFaceMed 6:25.

17. Reitzen SD, Babb JS, Lalwani AK (2009) Significance and reliability of the House-Brackmann grading system for regional facial nerve function. OtolaryngolHeadNeckSurg 140:154-158. 
18. Channer GA, Herman B, Telischi FF, Zeitler D, Angeli SI (2012) Management outcomes of facial nerve tumors: comparative outcomes with observation, CyberKnife, and surgical management. OtolaryngolHeadNeckSurg 147:525-530.

19. Perez R, Chen JM, Nedzelski JM (2005) Intratemporal facial nerve schwannoma: a management dilemma. OtolNeurotol 26:121-126.

20. Minovi A, Vosschulte R, Hofmann E, Draf W, Bockmuhl U (2004) Facial nerve neuroma: surgical concept and functional results. SkullBase 4:195-200.

21. Shirazi MA, Leonetti JP, Marzo SJ, Anderson DE (2007) Surgical management of facial neuromas: lessons learned. OtolNeurotol 28:958963.

22. Falcioni M, Russo A, Taibah A, Sanna M (2003) Facial nerve tumors. OtolNeurotol 24:942-947.

23. Friedman O, Neff BA, Willcox TO, Kenyon LC, Sataloff RT (2002) Temporal bone hemangiomas involving the facial nerve. OtolNeurotol 23:760-766.

24. Litrŭ CF, Gourg GP, Tamura M, et al (2007) Gamma knife surgery for facial nerve schwannomas. Neurosurgery 60:853-859.

25. Kida Y, Yoshimoto M, Hasegawa T (2007) Radiosurgery for facial schwannoma. J Neurosurg 106:24-29.

26. Nishioka K, Abo D, Aoyama H, et al. (2009) Stereotactic radiotherapy for intracranial nonacousticschwannomas including facial nerve schwannoma. Int J RadiatOncolBiolPhys 75:1415-1419.

\section{Corresponding Author:}

\section{KH. Diab}

Federal Research Clinical Center of Otorhinolaryngology of Federal Medical Biological Agency of Russia, (Director - Professor N. A. Daikhes),

123182, Moscow, Russia

E-mail: hasandiab@mail.ru 